\title{
Attitudes of general practitioners to a novel gene-nutrient interaction and its implications for blood pressure management: Preliminary findings
}

\author{
N. Canning, G. Horigan, B. Whelan, H. McNulty, J. J. Strain and M. Ward \\ Northern Ireland Centre for Food and Health, University of Ulster, Coleraine, BT52 1SA, UK
}

Hypertension is a major risk factor for cardiovascular disease (CVD) and is estimated to account for 14\% of global mortality. The C677T polymorphism in the methylenetetrahydrofolate reductase (MTHFR) gene is associated with hypertension. In a recent study at this centre, intervention with the recommended dietary level $(1.6 \mathrm{mg} /$ day) of riboflavin, an essential co-factor (in the form of FAD) for MTHFR, significantly lowered blood pressure (BP) in CVD patients homozygous for this polymorphism (TT genotype) ${ }^{(1,2)}$. Further confirmation of the BP-lowering effect of riboflavin in this genotype group was subsequently demonstrated in a 4-year follow-up study of these patients ${ }^{(3)}$. The attitude of general practitioners (GPs) to this novel gene-nutrient interaction is currently unknown, but is of importance in the wider translation of this research given that they have a key role to play in BP management. The aim therefore of this study was to explore the level of interest of GPs in this novel treatment option for hypertension and in particular their attitude to recommending riboflavin as a targeted treatment for elevated BP specifically in patients with the MTHFR 677TT genotype. A questionnaire was designed, and following initial feedback from a pilot study, a final version was generated. This was deliberately short (i.e. one-page) but links to the relevant scientific publications ${ }^{(2,3)}$ were made available so that respondents had access to full details as required. Questionnaires were distributed via email to a sample of GPs practising in the Western Health and Social Care Trust, Northern Ireland.

Based on an initial return of 53 questionnaires, descriptive statistics and cross tabulations (SPSS) were used to examine responses. In terms of recommending low dose riboflavin for the non-targeted treatment of elevated BP, the majority of respondents indicated that they would $(28 \%)$ or possibly would $(47 \%)$ consider this option (Fig. 1). When asked about riboflavin in the targeted treatment of hypertension in patients with the TT genotype, a much greater number of GPs responded that they would (45\%) or possibly would (51\%) consider this treatment, with only $4 \%$ responding that they would not (Fig. 2). Of note, $51 \%$ of GPs $(n=27)$ would be willing to have their patients genetically screened for this polymorphism, while a further $47 \%(n=25)$ responded 'possibly' to that particular question. Encouragingly, $51 \%(n=27)$ of respondents were willing to receive information regarding a future related study, and of these, a number agreed that there 'may' $(26 \%)$ or 'may possibly' $(23 \%)$ be a role for this novel treatment as part of their routine management of BP.

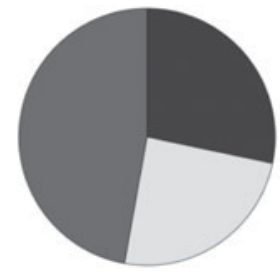

Figure 1: Would you consider riboflavin as a non-targeted treatment option?
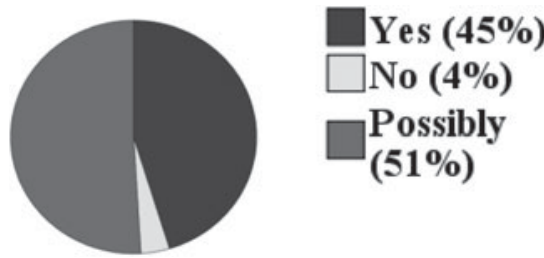

Figure 2: Would you consider riboflavin as a targeted treatment option (i.e. for TT genotype?)

These preliminary results indicate a positive attitude of GPs to this novel treatment option for hypertension, and particularly as a targeted treatment strategy for patients with the MTHFR 677TT genotype. Confirmation of these findings in a larger sample is warranted.

1. Heux et al. (2004) Hypertens Res 27, 663-7.

2. Horigan et al. (2010) J Hypertens 28, 478-86.

3. Wilson et al. (2012) Am J Clin Nutr 95, 766-72. 